\title{
Short duration of neutralizing antibody titers after pre-exposure rabies vaccination with suckling mouse brain vaccine
}

\section{C.R. Zanetti ${ }^{1}$, C.A. Consales ${ }^{2}$, A.C. Rodrigues-da-Silva², Y.K. Toyoshima ${ }^{3}$ and O.A.C. Pereira ${ }^{2}$}

\author{
1Disciplina de Imunologia, Departamento de Microbiologia e Parasitologia, \\ Centro de Ciências Biológicas, U niversidade Federal de Santa Catarina, \\ Florianópolis, SC, Brasil \\ ${ }^{2}$ Setor de Sorologia, Instituto Pasteur de São Paulo, São Paulo, SP, Brasil \\ ${ }^{3}$ Secretaria da Ação Social de Jaboticabal, Jaboticabal, SP, Brasil
}

\section{Correspondence \\ C.R. Zanetti \\ Disciplina de Imunologia \\ Departamento de Microbiologia e \\ Parasitologia, CCB, UFSC \\ 88040-900 Florianópolis, SC \\ Brasil \\ Fax: + 55-48-331-9258 \\ E-mail: zanetti@ccb.ufsc.br}

Publication supported by FAPESP.

Received February 4, 1998 Accepted July 7, 1998

\section{Abstract}

The human anti-rabies pre-exposure treatment currently used in Brazil, employing a $1-\mathrm{ml}$ dose of suckling mouse brain vaccine (SMBV) administered on days $0,2,4$ and 28, was compared to an alternative treatment with two $1 \mathrm{ml}$-doses on day 0 , and one $1 \mathrm{ml}$-dose injected on days 7 and 21. The latter induced higher virus-neutralizing antibody (VNA) titers on day 21. Both Brazilian rabies vaccines produced with PV or CVS rabies virus strains were tested. Two additional volunteer vaccinee groups, receiving the pre-exposure and the abbreviated postexposure schedules recommended by the WHO using cell-culture vaccine $(\mathrm{CCV})$ produced with PM rabies virus strain, were included as reference. The VNA were measured against both PV and CVS strains on days 21,42 and 180 by the cell-culture neutralization microtest. The PV-SMBV elicited higher seroconversion rates and VNA by day 21 than the CVS-SMBV. Both, however, failed to induce a long-term immunity, since VNA titers were $<0.5 \mathrm{IU} / \mathrm{ml}$ on day 180 , regardless of the schedule used. Cell-culture vaccine always elicited very high VNA on all days of collection. When serum samples from people receiving mouse brain tissue were titrated against the PV and CVS strains, the VNA obtained were similar, regardless of the vaccinal strain and the virus used in the neutralization test. These results contrast with those obtained with sera from people receiving PM-CCV, whose VNA were significantly higher when tested against the CVS strain.
The WHO Expert Committee on Rabies states that the utilization of brain tissue vaccines must be discouraged, and adds that countries where this kind of vaccine is still being used must standardize their immunization regimens under their own conditions (1). Since cell-culture vaccine (CCV) for human treatment is not available in the Brazilian Public Health Services, we have stud-

\section{Key words}

- Rabies

- Rabies vaccine

- Pre-exposure

- Suckling mouse brain vaccine ied different immunization schedules employing brain tissue vaccines $(2,3)$. In a field study carried out in 29 different localities in the State of São Paulo, we found that the preexposure anti-rabies immunization regimen currently used in Brazil (a 1-ml im dose of suckling mouse brain vaccine (SMBV) on days $0,2,4$ and 28) showed an elevated rate of unsatisfactory results, i.e., virus-neutral- 
izing antibody (VNA) titers <0.5 IU (4). These results contrasted with others obtained in well-controlled trials using the same regimen $(5,6)$. Several reasons, such as improper transportation, storage or administration of the vaccine (e.g., injections into the gluteus instead of the deltoid muscle), variable NIH potency among vaccine batches, and other local problems, were proposed as possible causes of unsatisfactory antibody titers. In addition, the human rabies vaccines produced in Brazil are prepared by two different laboratories, which propagate the virus using the nervous system of suckling mice (7) and use the PV or CVS strains. These vaccines are released with an NIH potency $\geq 1$. In the State of São Paulo, the vaccine available is the one produced with the CVS strain. Since the virus neutralization antibody test has been performed with the PV strain, a lack of complete cross-neutralization could be an additional factor contributing to the relatively low antibody levels obtained.

In the present study, the pre-exposure 02-4-28 schedule was compared to the 00-721 schedule, using SMBVs produced with either the PV or CVS strain. The 00-7-21 schedule is currently recommended by the WHO for post-exposure treatment with CCV. The elicited VNA were titrated against the two vaccinal virus strains used in Brazil. Moreover, two groups receiving pre- or postexposure regimens with the Pittman-Moore rabies vaccine cultivated in VERO cells were included as reference.

A total of 106 veterinary students who had never received rabies vaccine, ranging in age from 17 to 22 years, gave informed consent to participate voluntarily in the study. Six groups of students alphabetically organized to attend dissection practices were assigned to receive rabies vaccine according to one of the following schedules. Group 02-4-28 PV-SMBV: 18 subjects who received the Fuenzalida and Palacios vaccine produced with the PV strain, administered on days $0,2,4$ and 28; group 00-7-21 PV-
SMBV: 19 subjects who received the Fuenzalida and Palacios vaccine produced with the PV strain, administered on days 0 (two doses), 7 and 21; group 0-2-4-28 CVSSMBV: 23 subjects who received the Fuenzalida and Palacios vaccine produced with the CVS strain, administered on days 0 , 2, 4 and 28; group 00-7-21 CVS-SMBV: 26 subjects who received the Fuenzalida and Palacios vaccine produced with the CVS strain, administered on days 0 (two doses), 7 and 21; group 00-7-21 PM-CCV: 10 subjects who received a post-exposure schedule with the VERO cell-culture vaccine produced with the PM strain, administered on days 0 (two doses), 7 and 21; group 0-7-28 PM-CCV: 10 subjects who received a preexposure schedule with the same VERO cellculture vaccine produced with the PM strain, on days 0,7 and 28 . All doses were of $1.0 \mathrm{ml}$ and were injected into the deltoid region. Both Fuenzalida and Palacios vaccines currently produced in Brazil for human treatment were tested: PV-SMBV, a $2 \%$ UVinactivated suspension of suckling mouse brain infected with the PV strain, was provided by Instituto Butantan, São Paulo (lot No. 9403029 , NIH potency value $=1.5 \mathrm{IU} /$ dose) and CVS-SMBV, a $2 \%$ betapropiolactone-inactivated suspension of suckling mouse brain infected with the CVS strain, was provided by Instituto de Tecnologia do Paraná, Curitiba, PR (lot 049-93, NIH potency value $=1.4 \mathrm{IU} /$ dose) . The cell-culture vaccine used as reference, PM-CCV, was prepared with the PM strain in VERO cells, and was provided by Pasteur-Mérieux Sérums and Vaccins, Lyon, France (lot No. J1213, $\mathrm{NIH}$ potency value $\geq 2.5 \mathrm{IU} /$ dose).

Serum samples were collected on days 0 , 21, 42 and 180 after the first rabies vaccine injection. Virus-neutralizing antibodies against both the PV and CVS strains were submitted to the simplified fluorescence inhibition microtest as previously described (2). VNA is expressed as IU/ml, using as reference an equine hyperimmune serum 
adjusted to a concentration of $2.0 \mathrm{IU} / \mathrm{ml}$. The seropositivity of each group was estimated as the percentage of patients who developed VNA higher than $0.5 \mathrm{IU} / \mathrm{ml}$. Unfortunately, 14 of 18 volunteers in group 0-2-4-28 PVSMBV had to be excluded from the study before day 180 . The reason was that during a class, before study day 180 , those 14 subjects were exposed to an animal suspect of rabies, and were immediately treated with PM-CCV, invalidating serology results thereafter. The data regarding the differences among the VNA values for the 6 groups of volunteers were analyzed for statistical significance by analysis of variance (ANOVA) for repeated measures. The differences in seroconversion rates were analyzed by the chi-square test.

The results obtained with SMBVs are summarized in Table 1, which shows the percentage of seroconversion, the geometric mean titers (GMT), standard deviation (SD) and range of VNA against the CVS and PV strains. The results correspond to titers on days 21,42 and 180 . On day 0 , all serum samples showed titers $<0.16 \mathrm{IU} / \mathrm{ml}$ (data not shown). There were no significant differences on any collection day between VNA obtained against either PV or CVS strain, regardless of the vaccine schedule. Unfortunately, the difference between VNAs obtained for the CVS and PV strains could not be compared on day 180 , since it was only possible to evaluate 4 serum samples on that day.

The seroconversion rates were similar in all groups and on all collection days, except for the 0-2-4-28 CVS-SMBV group, which showed significantly lower rates on day 21 $(\mathrm{P}<0.01)$. The PV-SMBV schedules elicited higher VNA than the CVS-SMBV ones, regardless of the immunization schedule. The results obtained on days 21 and 42 showed that the suckling mouse brain vaccine produced with the PV strain performed better $(\mathrm{P}<0.01)$ than the one produced with the CVS strain, although both had a very similar
NIH potency titer. Actually, their relative potencies may not have been equivalent, since the NIH test has been shown to have very low reproducibility $(8,9)$.

The 00-7-21 schedules induced higher VNA titers than the 0-2-4-28 schedules by day 21, while the VNA induced by the latter schedules had a significant increase on day 42. The 00-7-21 schedule was able to elicit satisfactory VNA on day 21 in all but one vaccinee. Thus, we speculate whether this regimen could be used as a post-exposure prophylaxis, when the induction of an early antibody response is the goal. A reduction of the current post-exposure schedule used in Brazil, which consists of 13 doses associated with hyperimmune serum, or 10 doses when serum is not administered, would be of interest for several reasons. About 800,000 vaccine doses are administered each year, and the brain tissue vaccines may cause adverse neurological effects, mainly after administration of a large number of doses (10). Furthermore, the prolonged treatment is frequently abandoned, and, according to the Brazilian Health Ministry, an alarming 16\% of the post-exposure treatments are not concluded.

Pre-exposure regimens are intended to induce long-term immunity in persons who work under risk of contracting rabies. In this regard, both the 00-7-21 and the 0-2-4-28 schedules produced unsatisfactory results. Fifty-six percent or less of the vaccinees had VNA $<0.5 \mathrm{IU} / \mathrm{ml}$ on day 180 . These results are similar to those routinely observed at the Instituto Pasteur of São Paulo (Zanetti CR, unpublished data).

Both groups receiving cell-culture vaccine (Table 1) showed 100\% seroconversion rates on all collection days against both virus strains used in the neutralization test, except on day 0 when all serum samples showed titers $<0.16 \mathrm{IU} / \mathrm{ml}$ (data not shown). The titers obtained with the reduced post-exposure regimen (00-7-21) were significantly higher than those obtained with the pre- 
Table 1 - Seroconversion (seroc.) rates and antibody response to the PV and CVS rabies virus strains as a function of different vaccination schedules employing suckling mouse brain vaccines produced with PV and CVS rabies strains, and cell-culture vaccine produced with PM rabies strain.

aResults obtained when the PV strain was used in the neutralization test; bresults obtained when the CVS strain was used in the neutralization test; ' suckling mouse brain vaccine prepared with the PV rabies virus strain; ${ }^{d} 1$-ml dose of SMBV administered im on days 0, 2, 4 and 28; etwo 1-ml doses of SMBV administered im on day 0 and 1-ml dose administered on days 7 and 21; fsuckling mouse brain vaccine prepared with the CVS rabies virus strain; gcell-culture vaccine prepared with the PM rabies virus strain; ${ }^{h} 1-m l$ dose of CCV administered im on days 0,7 and $28 . \mathrm{GMT}=$ Geometric mean titer.

\begin{tabular}{|c|c|c|c|c|c|c|c|c|}
\hline \multirow[t]{3}{*}{ Vaccine } & \multirow[t]{3}{*}{ Schedule } & \multirow[t]{3}{*}{ Parameter } & \multicolumn{6}{|c|}{ Day after 1st dose } \\
\hline & & & \multicolumn{2}{|c|}{21} & \multicolumn{2}{|c|}{42} & \multicolumn{2}{|c|}{180} \\
\hline & & & $\mathrm{PV}$ & CVSb & PV & CVS & PV & CVS \\
\hline \multirow[t]{6}{*}{ PV-SMBVC } & $0-2-4-28^{d}$ & $\mathrm{~N}$ & 18 & 18 & 17 & 17 & 4 & 4 \\
\hline & & \% seroc. & 94.5 & 94.5 & 100 & 100 & 0 & 75 \\
\hline & & $\mathrm{GMT}$ & 2.47 & 1.63 & 6.56 & 6.43 & 0.22 & 0.32 \\
\hline & & SD & 4.25 & 3.93 & 4.27 & 4.22 & 0.08 & 0.12 \\
\hline & & Minimum & 0.47 & 0.32 & 2.67 & 2.25 & 0.17 & 0.25 \\
\hline & & Maximum & 17.93 & 17.93 & 15.08 & 15.08 & 0.34 & 0.50 \\
\hline \multirow[t]{6}{*}{ PV-SMBV } & $00-7-21^{e}$ & $\mathrm{~N}$ & 19 & 19 & 18 & 19 & 17 & 18 \\
\hline & & $\%$ seroc. & 100 & 100 & 100 & 100 & 40 & 40 \\
\hline & & GMT & 2.82 & 2.89 & 2.12 & 2.69 & 0.43 & 0.43 \\
\hline & & SD & 2.61 & 3.24 & 2.18 & 2.75 & 0.36 & 0.25 \\
\hline & & Minimum & 0.67 & 0.84 & 0.50 & 0.71 & 0.17 & 0.17 \\
\hline & & Maximum & 10.67 & 12.68 & 6.73 & 10.67 & 1.00 & 1.00 \\
\hline \multirow[t]{6}{*}{ CVS-SMBVf } & $0-2-4-28$ & $\mathrm{~N}$ & 23 & 23 & 21 & 21 & 16 & 16 \\
\hline & & \% seroc. & 21.7 & 30.5 & 100 & 95.2 & 56.3 & 50 \\
\hline & & $\mathrm{GMT}$ & 0.90 & 0.79 & 2.89 & 2.97 & 0.43 & 0.48 \\
\hline & & SD & 0.76 & 0.80 & 2.29 & 1.94 & 2.41 & 2.40 \\
\hline & & Minimum & 0.17 & 0.17 & 0.23 & 0.20 & 0.17 & 0.17 \\
\hline & & Maximum & 2.67 & 2.67 & 8.97 & 6.34 & 10.00 & 10.00 \\
\hline \multirow[t]{6}{*}{ CVS-SMBV } & $00-7-21$ & $\mathrm{~N}$ & 26 & 25 & 25 & 25 & 22 & 21 \\
\hline & & \% seroc. & 100 & 96 & 100 & 100 & 31.8 & 45.5 \\
\hline & & GMT & 2.19 & 1.88 & 2.15 & 2.27 & 0.42 & 0.49 \\
\hline & & SD & 4.96 & 3.10 & 1.30 & 1.61 & 0.52 & 0.50 \\
\hline & & Minimum & 0.59 & 0.47 & 0.67 & 0.71 & 0.17 & 0.17 \\
\hline & & Maximum & 25.36 & 15.08 & 5.33 & 5.66 & 2.00 & 2.00 \\
\hline \multirow[t]{6}{*}{ PM-CCVg } & $00-7-21^{e}$ & $\mathrm{~N}$ & 10 & 10 & 10 & 10 & 9 & 9 \\
\hline & & \% seroc. & 100 & 100 & 100 & 100 & 100 & 100 \\
\hline & & GMT & 29.21 & 43.31 & 25.39 & 35.96 & 6.28 & 8.94 \\
\hline & & SD & 26.00 & 33.16 & 20.03 & 23.17 & 12.38 & 14.45 \\
\hline & & Minimum & 16.67 & 25.00 & 16.67 & 25.00 & 2.00 & 4.00 \\
\hline & & Maximum & 100.00 & 133.33 & 83.00 & 100.00 & 32.00 & 43.50 \\
\hline \multirow[t]{6}{*}{ PM-CCV } & $0-7-28^{h}$ & $\mathrm{~N}$ & 10 & 10 & 10 & 10 & 10 & 10 \\
\hline & & \% seroc. & 100 & 100 & 100 & 100 & 100 & 100 \\
\hline & & GMT & 10.63 & 15.57 & 14.43 & 17.06 & 5.17 & 8.44 \\
\hline & & SD & 7.64 & 10.33 & 10.17 & 10.63 & 12.17 & 18.21 \\
\hline & & Minimum & 4.17 & 6.25 & 4.17 & 6.25 & 1.00 & 2.00 \\
\hline & & Maximum & 25.00 & 33.33 & 33.33 & 33.33 & 42.50 & 64.00 \\
\hline
\end{tabular}


exposure treatment (0-7-28) on day 21 $(\mathrm{P}<0.01)$, but not on subsequent days. Nevertheless, when CCV was used, the titers obtained with CVS were significantly higher than those obtained with the PV strain $(\mathrm{P}<0.01)$, except for 0-7-28 PM-CCV which, on day 21, showed similar VNA against both strains. These results agree with those observed by other authors (11) who have reported that, despite a common origin, the PV and PM strains are different when studied by neutralizing monoclonal antibody analysis. Molecular studies have also shown that CVS is much closer to PM than to PV (12).

The studies cited here indicate that, although the pre-exposure anti-rabies treatment currently used in Brazil elicits high seroconversion rates on day 21 , the response is short lasting. People receiving this kind of treatment should have a serum sample tested three months after treatment, a booster dose being administered when titers fall below $0.5 \mathrm{IU} / \mathrm{ml}$. Further studies will be needed to test the validity of this approach.

With respect to cell-culture vaccines, our results clearly confirm those reported by other investigators, who have shown very high VNA responses (13-15). Both the abbreviated post-exposure 00-7-21 schedule and the pre-exposure regimen induced an early and high antibody response. Whatever the schedule used and the collection day studied, the VNA obtained with cell-culture vaccine were always much higher than those obtained with suckling mouse brain vaccine.

The superior performance of rabies vaccines produced in cell culture emphasizes the need to use them instead of those produced in animal brain. It is evident that efforts should be made by health authorities worldwide to produce or purchase rabies cell-culture vaccines. Brain tissue rabies vaccines are still frequently used (16), and in Brazil alone almost 200,000 vaccinees receive suckling mouse brain vaccine each year, demanding approximately 800,000 annual doses, a production fully funded by the Government. In this scenario, the complete replacement of currently used vaccines by cell-culture vaccine would be very costly. An important goal to be reached is a reduction in indications for human treatment, achievable only through an improvement of the National Program of Rabies Prophylaxis, concerning mainly the capture of stray dogs and observation and confirmation of rabies diagnosis in aggressor animals. When these measures are rigorously implemented, a drastic reduction in the number of rabies vaccinees should be expected and the replacement of the Fuenzalida and Palacios vaccine by a more potent and safe, although more expensive, cell-culture vaccine may be envisaged.

\section{Acknowledgments}

The authors gratefully acknowledge the contribution of the volunteers of the School of Veterinary Medicine of Jaboticabal (UNESP), and also thank Mrs. Dolores Ayako Yoda, Mrs. Maria Aparecida da Silva, Mrs. Márcia Harder, Mrs. Maria das Graças Silva, and Mrs. Luciana Botelho Chaves for technical assistance.

\section{References}

1. WHO (1992). Expert Committee on Rabies. 8th report. Technical Report Series No. 824. World Health Organization, Geneva.

2. Favoretto SR, Carrieri ML, Tino MS, Zanetti CR \& Pereira OAC (1993). Simplified fluorescence inhibition microtest for the titration of rabies neutralizing antibodies. Revista do Instituto de Medicina Tropical de São Paulo, 35: 171-175.

3. Zanetti CR, Lee LM, Chaves LB, Rodrigues J L, Eleutério GC \& Pereira OAC (1995). Studies on human anti-rabies immunization in Brazil. Preliminary evalua- tion of the 00-7-21 schedule for human anti-rabies immunization, employing suckling mouse brain vaccine. Revista do Instituto de Medicina Tropical de São Paulo, 37: 353-356.

4. Zanetti CR, Chaves LB, Rodrigues Da Silva AC, Lee LM \& Pereira OAC (1995). Stud- 
ies on human anti-rabies immunization in Brazil. I - Evaluation of the 0-2-4-28 preexposure vaccination schedule under field conditions. Revista do Instituto de Medicina Tropical de São Paulo, 37: 349a-352a.

5. Chamelet ELB, Azevedo MP, Favoretto SR, Kerbrie SV \& Souza LTM (1982). Esquema reduzido de vacinação antirábica humana pré-exposição e avaliação de doses anuais de reforço. Revista de Saúde Pública de São Paulo, 16: 144-148.

6. Favoretto SR, Carrieri ML, Tino MS, Zanetti CR \& Pereira OAC (1993). Reduced schedule of human anti-rabies immunization with Fuenzalida and Palacios vaccine. Additional data. Revista do Instituto de Medicina Tropical de São Paulo, 35: 281-284.

7. Fuenzalida E \& Palacios R (1955). Un método mejorado en la preparación de la vacuna antirábica. Boletín del Instituto de Bacteriologia de Chile, 8: 3-10.

8. Barth R, Gross-Albenhausen E, J aeger O $\&$ Milcke $L$ (1981). The antibody-binding test, a useful method for quantitative determination of inactivated rabies virus antigen. J ournal of Biological Standardization, 9: 81-89.

9. Albas A, Mourão Fuches RM \& Frazatti Galina NM (1992). Termoestabilidade da vacina contra a raiva, tipo Fuenzalida \& Palacios, uso humano. Revista do Instituto de Medicina Tropical de São Paulo, 34: 27-31.

10. Held RJ \& Adaros LH (1972). Neurological disease in man following administration of suckling mouse brain anti-rabies vaccine. Bulletin of the World Health Organization, 46: 321-327.

11. Lafon M, Bourhy H \& Sureau P (1988). Immunity against the European bat rabies (Duvenhage) virus induced by rabies vaccine: an experimental study in mice. Vaccine, 6: 362-368.

12. Tordo N, Sacramento D \& Bourhy H (1996). The polymerase chain reaction (PCR) technique for diagnosis, typing and epidemiological studies of rabies. In:
Meslin F-X, Kaplan MM \& Koprowski H (Editors), Laboratory Techniques in Rabies. 4th edn. World Health Organization, Geneva.

13. Vodopija I, Sureau P \& Lafon M (1986). An evaluation of second generation tissue culture rabies vaccines for use in humans: a four vaccine comparative immunogenicity study using a pre-exposure vaccination schedule and an abbreviated 00-7-21 post-exposure schedule. Vaccine, 44: 245248.

14. Vodopija I, Sureau P \& Smerdel S (1988). Interaction of rabies vaccine with human rabies immunoglobulin and reliability of a 00-7-21 schedule application for post-exposure treatment. Vaccine, 6: 283-286.

15. Chavenet $P$, Sureau $P \&$ WaldnerCombernoux A (1989). Schéma simplifié pour la vaccination antirabique après exposition. Presse Médicale, 18: 813-815.

16. World Survey of Rabies for the year 1994 (No. 30), WHO/EMC/ZOO/96.3. World Health Organization, Geneva. 